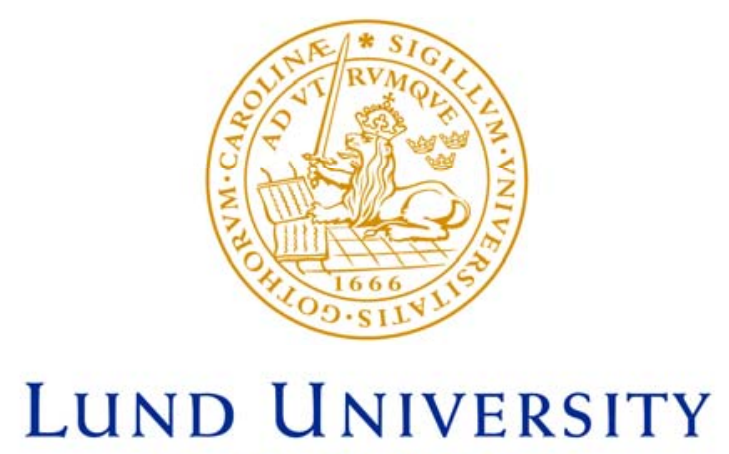

Faculty of Medicine

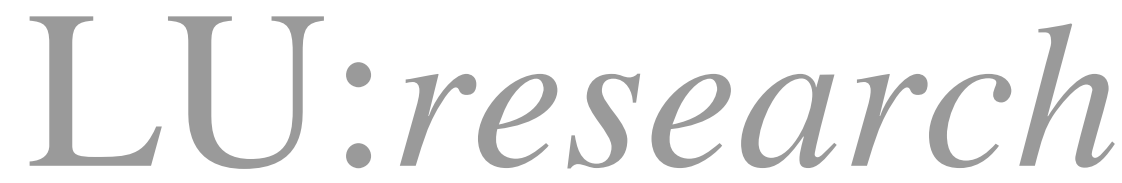

Institutional Repository of Lund University

This is an author produced version of a paper published in European journal of clinical microbiology \& infectious diseases: official publication of the European Society of Clinical Microbiology. This paper has been peer-reviewed but does not include the final publisher proof-corrections or journal pagination.

Citation for the published paper:

Riesbeck, K and Hammas, B.

"Comparison of an automated Borrelia indirect chemiluminescent immunoassay (CLIA) with

a VIsE/C 6 ELISA and Immunoblot.

Eur J Clin Microbiol Infect Dis, 2007, Vol: 26, Issue: 7, pp. 517-519.

http://dx.doi.org/ 10.1007/s10096-007-0329-x

Access to the published version may require journal subscription.

Published with permission from: Springer 
BRIEF REPORT

\title{
Comparison of an automated Borrelia indirect chemiluminescent immunoassay (CLIA) with a VlsE/C6 ELISA and Immunoblot
}

\author{
K. Riesbeck and B. Hammas
}

Keywords: Borrelia burgdorferi, Serology, ELISA, Chemiluminescence, Immunoblot

K. Riesbeck and B. Hammas

Medical Microbiology, Department of Laboratory Medicine

Malmö University Hospital, Lund University

20502 Malmö, Sweden

e-mail: kristian.riesbeck@med.lu.se 
The B. burgdorferi sensu lato complex consists of eleven different species [1]. B. burgdorferi sensu stricto are isolated from patients both in North America and Europe, whereas B. garinii and B. afzelii are mainly found in Europe. The complete genome of $B$. burgdorferi has been sequenced and recent years a growing body of knowledge on important surface proteins has accumulated [2]. Borrelia Outer surface protein C (OspC) [3] and Vmplike sequence expressed (VlsE) [4] are two plasmid encoded membrane proteins that both are immunodominant antigens. Furthermore, VlsE consists of one invariable region (IR6), which is extraordinary immunodominant $[5,6]$. Other antigens that have been useful in serology are p39 and p83 [7]. Proteins that only are expressed in vivo are, for example, protein G, BBA36, and BB0323 with to date unknown functions, and finally the factor $\mathrm{H}$ binding protein Crasp3.

Several different laboratory methods have been used for the detection of $B$. burgdorferi [for a review see 8]. The most utilized diagnostic procedures are based upon serological diagnosis of Borrelia, and many laboratories use a screening method such as ELISA followed by Immunoblots containing a panel of different specific Borrelia antigens. This two-tier approach is according to the current recommendations from CDC [9]. A combination of a higher incidence ( 8.2 cases per 100,000 population during 2002 in the US) of Lyme disease (LD) [1] and an increased awareness by the clinicians has led to the need for larger series of testing. Hence, the need for automatization and large-scale analyses is more important than ever. The objective of this study was to evaluate and compare an indirect chemiluminescent immunoassay (CLIA) with a conventional VlsE/C6 ELISA using a two-tier approach.

Sera from patients $(n=157)$ visiting outpatient clinics were analysed for Borrelia antibodies. The collection consisted of 72 males (ages 5-88 years) and 85 females (ages 1083). Patients visited their physicians because of anxiety about borrelia or suspicion of multisystem infection (mainly arthritis) or late borreliosis. Moreover, additional sera from patients $(n=11)$ that were referred to Dept. of Infectious Diseases (Malmö University Hospital) due to late borreliosis were also analysed. In addition to sera from healthy blood donors $(n=20)$, acute sera $(n=23)$ with high IgM titers against EBV, CMV, VZV, HSV, or finally parvovirus were included for comparison. An instrument analysing Borrelia immunoglobulin M and G by a two step sandwich CLIA (Liaison; DiaSorin, Salugia, Italy) was used [10]. The CLIA consisted of the recombinant antigens OspC (from the strain $B$. afzelii $\mathrm{Pko}$ ) and VlsE (from B. garinii $\mathrm{P} / \mathrm{Bi}$ ) bound to magnetic microparticles for the analysis of IgM and IgG, respectively. In addition, an IgG/ IgM combination ELISA using VlsE/C6 as 
antigen (Quick; Immunetics, Boston, MA) was included. Positive sera were analysed in Immunoblots (Borrelia EcoLine; Genzyme Virotech, Rüsselsheim, Germany). For IgM analysis, the antigen OspC, VlsE and p39 were included on the strips. To exclude EBV infection, the Capsid Antigen gp125 was also present. For analysis of IgG directed against borrelia, the antigens VlsE, p39, p83, BBA36, BBO323, Crasp3, and pG were used. Interpretation of the Immunoblots was done according to the manufacturer's instructions. Briefly, a borderline result consisted of one IgM-band (except OspC) or a VlsE IgG-band. A sample was considered positive when an OspC IgM-bands, $\geq$ two IgM-bands, or $\geq$ two IgGbands were detected. In addition, only one IgM-band together with only one IgG-band resulted in a positive result. Further interpretation of the Immunoblots is specified in Table 1. Finally, to exclude cross reactivity with Treponema pallidum, all positive sera were analysed with Serodia TPPA (Fujirebio, NJ).

The consecutive patient sera were tested for antibodies against Borrelia in both CLIA and VlsE/C6 ELISA. Based upon the clinical findings (when available) and Immunoblot analyses after the initial screening assays, $82.2 \%$ of the samples were considered true negatives and $17.8 \%$ true positives. Twenty-seven patient sera out of a total of 28 positive ones were detected with the CLIA and/ or VlsE/C6 ELISA, whereas both methods failed to detect one serum each as shown in Table 1. The failures were sera from two different patients. Four more sera were false positive with the VlsE/C6 ELISA (5.4\%) as compared to the CLIA (2.3\%). These patients' past medical histories revealed that seven (with symptoms and diagnoses such as erythema nodosum, herpes zoster virus, or swollen knees) out of ten patients did not have any signs of LD. However, as can be seen in Table 1, they were negative in Immunoblots. No significant differences were observed between the two test systems when sera from patients with mainly late borreliosis (neurological symptoms or arthritis) were analysed. All these patient sera were found positive in both methods. One of these patients was also positive for syphilis and was found to suffer from both infections. When data from the consecutive patient sera and neuroborreliosis patients $(n=168)$ were analysed, the sensitivity and specificity of the Liaison system was 0.975 (CI 0.886-0.999) and 0.977 (CI 0.949-0.984), respectively. In parallel, the Immunetics VlsE/C6 ELISA had the same sensitivity (0.975, CI 0.875-0.999), but a slightly lower specificity (0.946, CI 0.916-0.953). The positive and negative predictive values, calculated upon the 157 consecutive patient sera, were for the CLIA 0.932, respectively, 1.000, and for the ELISA 0.822, respectively, 1.000. Two patients with high IgM titers for EBV, respectively, CMV were found to be false 
positive in the Immunetics ELISA (as verified with the Immunoblot), but negative in the CLIA. Finally, when 20 healthy blood donors were analysed for borrelia antibodies, no major differences were found between the two methods; two and one sera were positive in the ELISA and CLIA, respectively. These three samples were also positive according to the Immunoblot criteria.

In contrast to the CLIA, the VlsE/C6 ELISA does not discriminate between IgG and IgM. In our material, five sera were positive for IgM only (and not IgG) in the CLIA, but negative in the ELISA. These samples were confirmed by the IgM Immunoblot. No false positive IgM samples were found with the CLIA, but one more patient serum was positive for IgM in the Immunoblot. Since the handling time is minimal, the CLIA is more cost-effective as compared to conventional ELISAs and thus is an alternative for large scale laboratories. In the light of that convalescence samples sometimes are required to prove LD [6], the CLIA is an affordable option. In conclusion, the Liaison Borrelia CLIA is a reliable screening test for automatization and is comparable with the VlsE/C6 ELISA. It is questionable, however, whether it is an advantage to detect IgM separately since a two-tier testing procedure including IgM and IgG Western Blots still is recommended [8, 9]. Future studies will reveal whether the Liaison CLIA will be a method without the requirement for two-tier testing. 


\section{References}

1. Centers for Disease Control and Prevention (2004) Lyme Disease-United States 20012002. Morb Mortal Wkly Rep 53:365-369

2. Rosa PA, Tilly K, Stewart PE (2005) The burgeoning molecular genetics of the Lyme disease spirochaete. Nat Rev Microbiol 3:129-143

3. Padula SJ, Dias F, Sampieri A, Craven RB, Ryan RW (1994) Use of recombinant OspC from Borrelia burgdorferi for serodiagnosis of early Lyme disease. J Clin Microbiol 32:1733-1738

4. Sung SY, McDowell JV, Marconi RT (2001) Evidence for the contribution of point mutations to VlsE variation and for apparent constraints on the net accumulation of sequence changes in VlsE during infection with Lyme disease spirochetes. $J$ Bacteriol $183: 5855-5861$

5. Lawrenz MB, Hardham JM, Owens RT et al (1999) Human antibody responses to VlsE antigenic variation protein of Borrelia burgdorferi. J Clin Microbiol 37:39974004

6. Philipp MT, Marques AR, Fawcett PT, Dally LG, Martin DS (2004) C6 test as an indicator of therapy outcome for patients with localized or disseminated Lyme borreliosis. J Clin Microbiol 41:4955-4960

7. Hauser U, Lehnert G, Wilske B (1999) Validity of interpretation criteria for standardized Western blots (immunoblots) for serodiagnosis of Lyme borreliosis based on sera collected throughout Europe. J Clin Microbiol 37:2241-2247

8. Aguero-Rosenfeld ME, Wang G, Schwartz I, Wormser GP (2005) Diagnosis of Lyme borreliosis. Clin Microbiol Rev 18:484-509

9. Centers for Disease Control and Prevention (1995) Recommendations for test performance and interpretation from the Second National Conference on Serologic Diagnosis of Lyme Disease. Morb Mortal Wkly Rep 44:590-591

10. Marangoni A, Sambri V, Accardo S et al (2006) A decrease in the immunoglobulin G antibody response against the VlsE protein of Borrelia burgdorferi sensu lato correlates with the resolution of clinical signs in antibiotic-treated patients with early Lyme disease. Clin Vaccine Immunol 13: 525-9 
Table 1 Results for the Liaison automated chemiluminescent immunoassay (CLIA) and the Immunetics VlsE/C6 ELISA used to detect Borrelia IgG and IgM antibodies in sera from 157 consecutive patients

\begin{tabular}{lrrrrrr}
\hline \multirow{2}{*}{$\begin{array}{l}\text { Result by Immunoblot } \\
\text { No. }\end{array}$} & \multicolumn{2}{c}{ ELISA } & & \multicolumn{2}{c}{ CLIA $^{\mathrm{b}}$} \\
\cline { 3 - 4 } & & $\begin{array}{l}\text { No. }(\%) \\
\text { positive }\end{array}$ & $\begin{array}{l}\text { No. (\%) } \\
\text { negative }\end{array}$ & & $\begin{array}{l}\text { No. }(\%) \\
\text { positive }\end{array}$ & $\begin{array}{l}\text { No. (\%) } \\
\text { negative }\end{array}$ \\
\hline Positive & 28 & $27(96.4)$ & $1(3.6)$ & & $27(96.4)$ & $1(3.6)$ \\
Negative & 10 & $7(5.4)$ & $122(94.6)$ & & $3^{\mathrm{c}}(2.3)$ & $126(97.7)$ \\
\hline
\end{tabular}

${ }^{a}$ Sera that were positive in either screening test were analysed with Immunoblots. One sample was borderline in the Immunoblot but judged as positive based upon the clinical findings (neuroborreliosis).

${ }^{\mathrm{b}}$ Sera that were equivocal $(n=4)$ were considered as positive and tested with Immunoblots.

${ }^{\mathrm{c}}$ These three sera were IgG positive only 